INPLASY

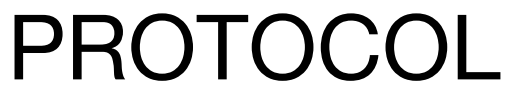

To cite: Guo et al. Efficacy and safety of percutaneous endoscopic lumbar discectomy via intervertebral approach for L5/S1 lumbar disc herniation: a systematic review and metaanalysis. Inplasy protocol 202170073. doi: 10.37766/inplasy2021.7.0073

Received: 23 July 2021

Published: 23 July 2021

Corresponding author:

Guo Tianci

2594576976@qq.com

Author Affiliation:

None

Support: National Natural

Science Found.

Review Stage at time of this submission: Data analysis.

Conflicts of interest:

None declared.

\section{Efficacy and safety of percutaneous endoscopic lumbar discectomy via intervertebral approach for L5/S1 lumbar disc herniation: a systematic review and meta-analysis}

Guo, T11; Feng, H2; Liu, A3; Chen, J4; Yu, W5.

Review question / Objective: P: L5/S1 patients with lumbar disc herniation. I: PEID. C: PETD. O: 1. Evaluation of clinical efficacy; VAS score; ODI score; 2. Safety evaluation; The operation time; Number of perspective; Intraoperative blood loss; Complication rate; The recurrence rate.

Condition being studied: L5/S1 lumbar disc herniation.

Information sources: Two researchers initially screened out the literature that might be needed according to the inclusion criteria, independently extracted the data (including the year of the literature author, the baseline data of the study object and related outcome indicators, etc.), and contacted the literature author to obtain more detailed information if necessary. When two researchers disagree, another author arbitrates.

INPLASY registration number: This protocol was registered with the International Platform of Registered Systematic Review and Meta-Analysis Protocols (INPLASY) on 23 July 2021 and was last updated on 23 July 2021 (registration number INPLASY202170073).

\section{INTRODUCTION}

Review question / Objective: P: L5/S1 patients with lumbar disc herniation. I: PEID. C: PETD. O: 1. Evaluation of clinical efficacy; VAS score; ODI score; 2. Safety evaluation; The operation time; Number of perspective; Intraoperative blood loss; Complication rate; The recurrence rate.

Condition being studied: L5/S1 lumbar disc herniation. 


\section{METHODS}

Participant or population: Patients with L5/ S1 lumbar disc herniation were included, and there were clear diagnostic criteria and surgical indications.

Intervention: The experimental group was treated with PEID.

Comparator: The control group was treated with PETD.

Study designs to be included: Type of study: Randomized controlled trial (RCT); Subjects: The included subjects were L5/S1 patients with lumbar disc herniation, and there were clear diagnostic criteria and surgical indications; Intervention: PEID was used in the experimental group, PETD was used in the control group; (4) Outcome indicators: VAS score, ODI index, operative time, intraoperative fluoroscopy times, complication rate and postoperative recurrence rate.

Eligibility criteria: (1) Literature that does not meet the inclusion criteria; (2) Duplicate published literature; (3) Literature where full text or original data cannot be obtained.

Information sources: Two researchers initially screened out the literature that might be needed according to the inclusion criteria, independently extracted the data (including the year of the literature author, the baseline data of the study object and related outcome indicators, etc.), and contacted the literature author to obtain more detailed information if necessary. When two researchers disagree, another author arbitrates.

Main outcome(s): VAS score and ODI index.

Quality assessment / Risk of bias analysis: The Cochrane handbook bias risk assessment tool was used to evaluate the quality of the included RCTS. The Cochrane handbook criteria included the following 7 items: (1) randomized allocation method; Distribution hides; (3) Blind evaluation of participants and patients; Results blind evaluation; (5) Integrity of the result data; Selective reporting; Other biases.

Strategy of data synthesis: Meta-analysis was performed on the data of the included literatures using RevMan $\mathbf{5 . 3}$ software. The fixed-effects model was used to estimate the combined effect size when the study heterogeneity was greater than 0.10 or $12<50 \%$ using the 2 test. Otherwise, a random effects model is used to estimate the combined effect size. When metaanalysis is unavailable, descriptive analysis will be performed. Subgroup analysis was performed according to study design type. When more than 10 literatures were included in a meta-analysis, a funnel plot was used to assess publication bias.

Subgroup analysis: None.

Sensitivity analysis: None.

Country(ies) involved: China.

Keywords: percutaneous endoscopic transforaminal discectomy, percutaneous endoscopic interlaminar discectomy, Lumbar disc herniation.

Contributions of each author:

Author 1 - Guo Tianci.

Author 2 - Feng Huichuan.

Author 3 - Liu Aifeng.

Author 4 - Chen Jixin.

Author 5 - Yu Weijie. 